PACS 31.15.A-; 32.30.-r

A. S. Chernyshev, O. L. Mykhailov, A. V. Tsudik, I. S. Cherkasova

Odessa State Environmental University, L’vovskaya str.15, Odessa-16, 65016, Ukraine

E-mail: veronevonor@gmail.com

\title{
RELATIVISTIC THEORY OF CALCULATION OF E1 TRANSITION AMPLITUDES, AND GAUGE INVARIANCE PRINCIPLE
}

\begin{abstract}
The combined relativistic energy approach and relativistic many-body perturbation theory with the zeroth order Dirac-Kohn-Sham one-particle approximation are used for estimating the energies and the E1 radiative transitions amplitudes (oscillator strengths) for the low-excited states of the francium. The comparison with available theoretical and experimental (compilated) data is performed. The important point is linked with an accurate accounting for the complex exchange-correlation (polarization) effect contributions and using the optimized one-quasiparticle representation in the relativistic many-body perturbation theory zeroth order that significantly provides a physically reasonable agreement between theory and precise experiment.
\end{abstract}

\section{Introduction}

The development of new directions in the field of laser, atomic physics, quantum electronics, etc., such as pulsed heating methods in research on controlled thermonuclear fusion, new laser schemes in VUV, X-ray spectral regions, astrophysical studies, etc., necessitates the solution of new classes of problems of atomic and laser physics at a fundamentally new level of theoretical consistency and accuracy. Significant progress in the development of experimental research methods, in particular, a significant increase in the intensity and quality of laser radiation, the use of accelerators, heavy ion colliders, sources of synchrotron radiation and, as a result, the possibility of studying more and more energy processes, stimulates the development of new theoretical methods in the theory of heavy atoms calculation of their characteristics, in particular, radiation and autoionization ones [1-10].

However, a study of the spectral characteristics of heavy atoms and ions in the Rydberg states has to be more complicated as it requires a necessary accounting for the relativistic, exchangecorrelations effects and possibly the QED corrections for superheavy atomic systems. The simultaneous correct accounting of relativistic, quantum electrodynamic (QED), and many- particle correlation effects is essential [1-10]. The results of calculating the characteristics of atomic processes based on modern theoretical methods often differ several times.

The difference in the values of the transition amplitudes, the oscillator strengths, and the radiation widths for heavy atoms using various expressions for the photon propagator reaches $5-30 \%$ (we are essentially talking about the nonfulfillment of the principle of gauge invariance when calculating physical quantities) [11-18]. From the point of view of applications for the majority of the most important atomic systems, there is very often partially or completely missing information on their energy, radiation or/ and autoionization characteristics (heavy atoms, atoms of alkaline-earth elements, lanthanides and actinides).

In this paper the combined relativistic energy approach and relativistic many-body perturbation theory with the zeroth order DiracKohn-Sham 1-particle approximation [2,19] are used for are used for estimating the energies and the E1 radiative transitions amplitudes (oscillator strengths) for some low-excited states of the francium atom and studying an effect of the gauge invariance on the transition amplitude values for heavy atoms on example of the francium. 


\section{The theoretical method}

In Refs. [2,18-22] the fundamentals of the relativistic many-body PT formalism have been in detail presented, so further we are limited only by the novel elements. Let us remind that the majority of complex atomic systems possess a dense energy spectrum of interacting states. In Refs. [10-12] there is realized a field procedure for calculating the energy shifts $\Delta \mathrm{E}$ of degenerate states, which is connected with the secular matrix $M$ diagonalization. The whole calculation of the energies and decay probabilities of a non-degenerate excited state is reduced to the calculation and diagonalization of the $M$. The complex secular matrix $M$ is represented in the form:

$$
M=M^{(0)}+M^{(1)}+M^{(2)}+M^{(3)} .
$$

where $M^{(0)}$ is the contribution of the vacuum diagrams of all order of PT, and $M^{(1)}, M^{(2)}, M^{(3)}$ those of the one-, two- and three-QP diagrams respectively. The diagonal matrix $M^{(1)}$ can be presented as a sum of the independent 1QP contributions. The optimized 1-QP representation is the best one to determine the zeroth approximation. In the relativistic energy approach, which has received a great application during solving numerous problems of atomic, molecular and nuclear physics (e.g., see Refs. [21-27]), the imaginary part of electron energy shift of an atom is directly connected with the radiation decay possibility (transition probability). An approach, using the Gell-Mann and Low formula with the QED scattering matrix, is used in treating the relativistic atom. The total energy shift of the state is usually presented in the form:

$$
\Delta \mathrm{E}=\operatorname{Re} \Delta \mathrm{E}+\mathrm{i} \Gamma / 2
$$

where $\Gamma$ is interpreted as the level width, and the decay possibility $\mathrm{P}=\Gamma$. The imaginary part of electron energy of the system, which is defined in the lowest order of perturbation theory as $[10,11]$ :

$$
\min \Delta E(B)=-\frac{e^{2}}{4 \pi} \sum_{\substack{\alpha>n>f \\ \alpha<n \leq f]}} V_{\alpha n \alpha n}^{\left|\omega_{\alpha n}\right|},
$$

where $(\alpha>n>f)$ for electron and $(\alpha<n<f)$ for vacancy. The matrix element is determined as follows:

$V_{i j k l}^{|\omega|}=\iint d r_{1} d r_{2} \Psi_{i}^{*}\left(r_{1}\right) \Psi_{j}^{*}\left(r_{2}\right) \frac{\sin |\omega| r_{12}}{r_{12}}\left(1-\alpha_{1} \alpha_{2}\right) \Psi_{k}^{*}\left(r_{2}\right) \Psi_{l}^{*}\left(r_{1}\right)$

where $\omega_{i j}$ is the transition frequency; $\alpha_{i}, \alpha_{j}$ are the Dirac matrices. The separated terms of the sum in (1) represent the contributions of different channels and a probability of the dipole transition

Naturally, the physical values should not depend on the calibration of the photonic propagator. In general form, it can be written as

$$
\begin{gathered}
D=D_{T}+C \cdot D_{L}, \\
D_{T}=\frac{\delta_{\mu v}}{k_{0}^{2}-k^{2}}, \\
D_{L}=\frac{k_{\mu} k_{v}}{k_{0}^{2}-k^{2}}
\end{gathered}
$$

where the term $D_{T}$ is corresponding to exchange by transverse photons, $D_{L}$ - longitudinal ones, $\mathrm{C}$ is the gauge constant. contribution of the main exchange-correlation (the second and higher orders of the atomic perturbation theory or fourth etc of the QED perturbation theory) diagrams to imaginary part of an electron energy shift looks like [11]:

$$
\begin{aligned}
& \operatorname{Im} E_{\text {ninv }}\left(\alpha-s \mid A_{d}\right)=-C \frac{e^{2}}{4 \pi} \iiint \int d r_{1} d r_{2} d r_{3} d r_{4} \\
& \sum\left(\frac{1}{\omega_{m n}+\omega_{\alpha_{s}}}+\frac{1}{\omega_{m n}-\omega_{\alpha_{s}}}\right) \Psi_{\alpha}^{+}\left(r_{1}\right) \Psi_{m}^{+}\left(r_{2}\right) \Psi_{s}^{+}\left(r_{3}\right) .
\end{aligned}
$$

$$
\cdot \Psi_{n}^{+}\left(r_{4} \chi 1-\alpha_{1} \alpha_{2}\right) / r_{\mathbf{2}} \cdot\left\{\left[\left(\alpha_{3} \alpha_{4}-\left(\alpha_{3} n_{3} \chi \alpha_{4} n_{3}\right) / r_{3} .\right.\right.\right.
$$$$
\sin \left[\omega_{\alpha_{n}}\left(r_{\mathrm{D}}+r_{\mathrm{3}}\right)+\omega_{\alpha_{n}} \cdot \cos \left[\omega_{\alpha_{n}}\left(r_{\mathrm{L}}+r_{\mathbf{3}}\right)\right]\left(1+\left(\alpha_{3} n_{3}\right)\right.\right.
$$$$
\left.\left.\left.\left(\alpha_{4} n_{3}\right)\right)\right]\right\} \Psi_{m}\left(r_{3}\right) \Psi_{\alpha}\left(r_{4}\right) \Psi_{n}\left(r_{2}\right) \Psi_{s}\left(r_{1}\right)
$$

Expression (6) can be represented as an a sum:

$$
\Sigma\left\langle\alpha m\left|W_{1}\right| \boldsymbol{s}\right\rangle\left\langle\boldsymbol{s}\left|W_{2}\right| m \alpha\right\rangle /\left(\omega_{m} \pm \omega_{\alpha S}\right)
$$

with (4) different operator combinations $W_{l}$, $W_{2}$. The sum over $n$ can be calculated by the 
method of differential equations. The index $m$ numbers a finite number of states occupied in the core and the state of the real continuum. The continuum-related part describes the vacuum polarization of the electron field and leads to divergent integrals in the non-renormalizable theory. Its contribution to the main contribution has an additional order of smallness $\left(a Z^{2}\right)$. The minimization of the density functional $\operatorname{Im} d E$ leads to the integral differential equation for the $r_{\mathrm{c}}$, that can be numerically solved. This step allows to determine the optimization parameter $b$. In Ref. [11] the authors elaborated a simplified computational procedure.

The contribution of the main exchange-correlation (the second and higher orders of the atomic perturbation theory or fourth etc ones of the QED perturbation theory) to imaginary part of an electron energy shift is determined by the polarizability of an atomic core, which is related to the electronic core density $r_{c}$. The expression (6) can be represented an a functional of the density $r_{c}$.

Under calculating the matrix elements (2) one should use the expansion for potential $\sin |\mathrm{w}| \mathrm{r}_{12} / \mathrm{r}_{12}$ on spherical functions as follows $[10,11]$ :

$\frac{\sin |\omega| r_{12}}{r_{12}}=\frac{\pi}{2 \sqrt{r_{1} r_{2}}} \sum_{\lambda=0}^{\infty}(\lambda) J_{\lambda+1 / 2}\left(|\omega| r_{1}\right) J_{\lambda+1 / 2}\left(|\omega| r_{2}\right) P_{\lambda}\left(\hat{\left.\cos r_{1} r_{2}\right)}(\right.$

where $J$ is the Bessel function of first kind and $(1)=21+1$. Substitution of the expansion (5) to matrix element of interaction gives as follows [14]:

$$
\begin{gathered}
V_{1234}^{\omega}=\left[\left(j_{1}\right)\left(j_{2}\right)\left(j_{3}\right)\left(j_{4}\right)\right]^{1 / 2} \sum_{\mu}(-1)^{\mu}\left(\begin{array}{cc}
j_{1} j_{3} & \lambda \\
m_{1}-m_{3} & \mu
\end{array}\right) \times \\
\times \operatorname{Im}\left\{Q_{\lambda}^{Q u l}(1234)+Q_{\lambda}^{B}(1234],\right.
\end{gathered}
$$

where $j_{i}$ is the total single electron momentums, $m_{i}$ - the projections; $Q^{Q u l}$ is the Coulomb part of interaction, $Q^{B r}$ - the Breit part. Their detailed definitions are presented in Refs. [10$11,18,19]$. The relativistic wave functions are calculated by solution of the Dirac equation with the potential, which includes the "outer electron- ionic core" potential and exchange-polarization potential [20]. In fact, we realize the procedure of optimization of relativistic orbitals base. The main idea is based on using ab ini- tio optimization procedure, which is reduced to minimization of the gauge dependent multielectron contribution $\operatorname{ImD} E_{n i n v}$ of the lowest QED PT corrections to the radiation widths of atomic levels. According to [11, 18], "in the fourth order of QED PT (the second order of the atomic PT) there appear the diagrams, whose contribution to the $\operatorname{ImD} E_{\text {ninv }}$ accounts for correlation effects and this contribution is determined by the electromagnetic potential gauge (the gauge dependent contribution)". The accurate procedure for minimization of the functional $\mathrm{ImdE}_{\text {ninv }}$ leads to the Dirac-Kohn-Sham-like equations for the electron density that are numerically solved by the Runge-Cutta standard method It is very important to know that the regular realization of the total scheme allows to get an optimal set of the 1QP functions and more correct results in comparison with so called simplified one, which has been used in Refs. [11-13] and reduced to the functional minimization using the variation of the correlation potential parameter $b$. Other details can be found in Refs. [11,18,19,29].

The adequate, precise computation of radiative parameters of the heavy Rydberg alkali-metal atoms within relativistic perturbation theory requires an accurate accounting for the multielectron exchange-correlation effects (including polarization and screening effects, a continuum pressure etc). These effects within our approach are treated as the effects of the perturbation theory second and higher orders. Using the standard Feynman diagrammatic technique one should consider two kinds of diagrams (the polarization and ladder ones), which describe the polarization and screening exchange-correlation effects. The detailed description of the polarization diagrams and the corresponding analytical expressions for matrix elements of the polarization interelectron interaction (through the polarizable core of an alkali atom) potential is presented in Refs. [2,18,19,29].

An effective approach to accounting for the polarization diagrams contributions is in adding the effective two-quasiparticle polarizable operator into the perturbation theory first order matrix elements. In Ref. [10] the corresponding non-relativistic polarization functional has been derived. More correct relativistic expression has 
been presented in the Refs. [2,18] and used in our theory.

The corresponding two-quasiparticle polarization potential looks as follows: (10a)

$$
\begin{aligned}
& V_{p o l}^{d}\left(r_{1} r_{2}\right)=X\left\{\int \frac{d r^{\prime}\left(\rho_{c}^{(0)}\left(r^{\prime}\right)\right)^{1 / 3} \theta\left(r^{\prime}\right)}{\left|r_{1}-r^{\prime}\right| \cdot\left|r^{\prime}-r_{2}\right|}-\right. \\
& \left.-\int \frac{d r^{\prime}\left(\rho_{c}^{(0)}\left(r^{\prime}\right)\right)^{1 / 3} \theta\left(r^{\prime}\right)}{\left|r_{1}-r^{\prime}\right|} \int \frac{d r^{\prime \prime}\left(\rho_{c}^{(0)}\left(r^{\prime \prime}\right)\right)^{1 / 3} \theta\left(r^{\prime \prime}\right)}{\left|r^{\prime \prime}-r_{2}\right|} /\left\langle\left(\rho_{c}^{(0)}\right)^{1 / 3}\right\rangle\right\}
\end{aligned}
$$

$$
\begin{array}{r}
\left\langle\left(\rho_{c}^{(0)}\right)^{1 / 3}\right\rangle=\int d r\left(\rho_{c}^{(0)}(r)\right)^{1 / 3} \theta(r), \\
\theta(r)=\left\{1+\left[3 \pi^{2} \cdot \rho_{c}^{(0)}(r)\right]^{2 / 3} / c^{2}\right\}^{1 / 2},
\end{array}
$$

where $\rho_{c}^{0}$ is the core electron density (without account for the quasiparticle), $\mathrm{X}$ is numerical coefficient, $\mathrm{c}$ is the light velocity. The contribution of the ladder diagrams (these diagrams describe the immediate interparticle interaction) is summarized by a modification of the perturbation theory zeroth approximation mean-field central potential (look [2,18]), which includes the screening (anti-screening) of the core potential of each particle by the two others. All computing was performed with using the modified PC code "Superatom-ISAN" (version 93).

\section{Results and conclusion}

We applied the above described approach to compute the oscillator strengths (reduced dipole matrix elements) for a number of transitions in spectra of the heavy alkali atoms and corresponding ions.

As an illustration we present below the data for francium. In Table 1 there are listed the theoretical reduced dipole matrix elements for a number of transitions, computed within: i) relativistic Hartree-Fock (RHF) method $[6]$, ii) the empirical relativistic model potential method (ERMP) [7], iii) the relativistic singledouble (SD) method in which single and double excitations of the Dirac-Hartree-Fock (DHF) wave function are included to all orders of perturbation theory [8] and iv) our data.

Let us note that the precise experimental data for the francium $7 \mathrm{p}_{1 / 2,3 / 2}-7 \mathrm{~s}$ transition are as follows: $7 \mathrm{p}_{1 / 2}-7 \mathrm{~s}=4.277$ and $7 \mathrm{p}_{3 / 2}-7 \mathrm{~s}=5.898$ [8]. The important features of the approach used are using the optimized one-particle representation and an effective taking into account the exchange-correlation (including the core polarization) effects (see Refs. [2,18-20,30]).

Really, as it is indicated in Ref. [8], the semiempirical values agree with the ab initio SD calculations to better than $1 \%$ with the exceptions of the $7 \mathrm{~s}-$ $8 \mathrm{p}$ and $7 \mathrm{~s}-9 \mathrm{p}$ transitions, where contributions from correlation corrections are very large. The most important conclusions relate to an effect of the gauge invariance on the transition amplitude values.

An estimate of the gauge-non-invariant contributions (the difference between the oscillator strengths values calculated with using the transition operator in the form of "length" G1 and "velocity" G2) is about $0.1 \%$. The theoretical data, obtained with using the different photon propagator gauges (Coulomb and Babushkin ones) are practically equal.

Table 1.

\section{Theoretical reduced dipole matrix elements for a set of Fr transitions}

\begin{tabular}{|c|c|c|}
\hline Transition & i: RHF & ii: ERMP \\
\hline $7 \mathrm{p}_{1 / 2}-7 \mathrm{~s}_{1 / 2}$ & $\begin{array}{r}4.279 \\
4.304\end{array}$ & - \\
\hline $8 \mathrm{p}_{1 / 2}-7 \mathrm{~s}_{1 / 2}$ & $\begin{array}{l}0.291 \\
0.301\end{array}$ & 0.304 \\
\hline $9 \mathrm{p}_{1 / 2}-7 \mathrm{~s}_{1 / 2}$ & - & 0.096 \\
\hline $7 \mathrm{p}_{3 / 2}-7 \mathrm{~s}_{1 / 2}$ & 5.894 & - \\
\hline $8 \mathrm{p}_{3 / 2}-7 \mathrm{~s}_{1 / 2}$ & 0.924 & 0.908 \\
\hline $9 \mathrm{p}_{3 / 2}-7 \mathrm{~s}_{1 / 2}$ & - & 0.420 \\
\hline Transition & iii: SD- & iv: Our data \\
\hline $7 \mathrm{p}_{1 / 2}-7 \mathrm{~s}_{1 / 2}$ & 4.256 & $4.275(\mathrm{G} 1)$ \\
& & $4.277(\mathrm{G} 2)$ \\
\hline
\end{tabular}




\begin{tabular}{|c|c|c|}
\hline $8 \mathrm{p}_{1 / 2}-7 \mathrm{~s}_{1 / 2}$ & 0.327 & 0.339 \\
& 0.306 & \\
\hline $9 \mathrm{p}_{1 / 2}-7 \mathrm{~s}_{1 / 2}$ & 0.110 & 0.092 \\
\hline $7 \mathrm{p}_{3 / 2}-7 \mathrm{~s}_{1 / 2}$ & 5.851 & 5.891 \\
\hline $8 \mathrm{p}_{3 / 2}-7 \mathrm{~s}_{1 / 2}$ & 0.934 & 0.918 \\
\hline $9 \mathrm{p}_{3 / 2}-7 \mathrm{~s}_{1 / 2}$ & 0.936 & 0.426 \\
\hline
\end{tabular}

\section{References}

1. Grant, I. Relativistic Quantum Theory of Atoms and Molecules. Oxford, 2007.

2. Glushkov, A., Khetselius, O., Svinaren-ko, A., Buyadzhi, V. Spectroscopy of autoionization states of heavy atoms and multiply charged ions. Odessa: 2015.

3. Buyadzhi, V. Laser multiphoton spectroscopy of atom embedded in Debye plasmas: multiphoton resonances and transitions. Photoelectronics. 2015, 24, 128-133.

4. Khetselius, O.Yu. Relativistic perturbation theory calculation of the hyperfine structure parameters for some heavy-element isotopes. Int. J. Quant. Chem. 2009, 109, 3330-3335.

5. Chernyakova, Y., Ignatenko, A., Vitavetskaya, L.A. Sensing the tokamak plasma parameters by means high resolution $\mathrm{x}$-ray theoretical spectroscopy method: new scheme. Sensor Electr. and Microsyst. Techn. 2004, 1, 20-24.

6. Dzuba, V.A., Flambaum, V.V., Sushkov, O.P. Calculation of energy levels, E1 transition amplitudes, and parity violation in $\mathrm{Fr}$ Phys. Rev. A. 1995, 51, 3454.

7. Marinescu, M., Vrinceanu, D., Sadeghpour, $H$. Radiative transitions and van der Waals coefficients for francium. Phys. Rev. A. 1998, 58, R4259.

8. Safronova, U., Johnson, W., Derevianko, A. Relativistic many-body calculations of energy levels, hyperfine constants, electricdipole matrix elements, and static polarizabilities for alkali-metal atoms. Phys. Rev. A. 1999, 60, 4476 .

9. Ivanov, L.N., Ivanova, E.P., Aglitsky, E.
Modern trends in the spectroscopy of multicharged ions. Phys. Rep. 1988, 166.

10. Svinarenko, A., Khetselius, O., Buyadzhi, V., Florko, T., Zaichko,P., Ponomarenko, E. Spectroscopy of Rydberg atoms in a Blackbody radiation field: Relativistic theory of excitation and ionization. J. Phys.: Conf. Ser. 2014, 548, 012048.

11. Glushkov, A.V., Ivanov, L.N. Radiation decay of atomic states: atomic residue polarization and gauge noninvariant contributions. Phys. Lett. A 1992, 170, 33-36.

12. Glushkov A.V., Ivanov, L.N. DC strong-field Stark effect: consistent quantum-mechanical approach. J. Phys. B: At. Mol. Opt. Phys. 1993, 26, L379-386.

13. Glushkov, A. Spectroscopy of atom and nucleus in a strong laser field: Stark effect and multiphoton resonances. J. Phys.: Conf. Ser. 2014, 548, 012020

14. Glushkov, A. Spectroscopy of coope-rative muon-gamma-nuclear processes: Energy and spectral parameters J. Phys.: Conf. Ser. 2012, 397, 012011.

15. Khetselius, O.Yu. Quantum Geometry: New approach to quantization of quasistationary states of Dirac equation for superheavy ion and calculating hyperfine structure parameters. Proc. Int. Geometry Center. 2012, 5(34), 39-45.

16. Danilov, V., Kruglyak, Y., Pechenaya, V. The electron density-bond order matrix and the spin density in the restricted CI method. Theor. Chim. Act. 1969, 13(4), 288-296.

17. Kruglyak, Yu. Configuration interaction in the second quantization representation: basics with application up to full CI. ScienceRise. 2014, 4(2), 98-115.

18. Glushkov, A.V. Relativistic and correlation effects in spectra of atomic systems. Astroprint: Odessa, 2006.

19. Khetselius, O.Yu. Hyperfine structure of atomic spectra.- Odessa: Astroprint, 2008

20. Glushkov, A., Buyadzhi, V., Svinarenko, A., Ternovsky, E. Advanced relativistic energy approach in electron-collisional spectroscopy of multicharged ions in plasma. Concepts, Methods, Applications of Quantum 
Systems in Chemistry and Physics (Springer). 2018, 31, 55-69.

21. Dubrovskaya, Yu., Khetselius, O.Yu., Vitavetskaya, L., Ternovsky, V., Serga, I. Quantum chemistry and spectroscopy of pionic atomic systems with accounting for relativistic, radiative, and strong interaction effects. Adv. Quantum Chem. 2019, 78, 193222.

22. Khetselius, O.Yu., Glushkov, A.V., Dubrovskaya, Yu., Chernyakova, Yu., Ignatenko, A., Serga, I., Vitavetskaya, L. Relativistic quantum chemistry and spectroscopy of exotic atomic systems with accounting for strong interaction effects. In Concepts, Methods and Applications of Quantum Systems in Chem. and Phys. Springer. 2018, 31, 71.

23. Buyadzhi, V.V., Chernyakova, Yu.G., Antoshkina, O., Tkach, T. Spectroscopy of multicharged ions in plasmas: Oscillator strengths of Be-like ion Fe. Photoelectronics. 2017, 26, 94-102.

24. Malinovskaya, S.V., Dubrovskaya, Yu.V., Zelentzova, T.N. The atomic chemical environment effect on the $\mathrm{b}$ decay probabilities: Relativistic calculation. Herald of Kiev Nat. Univ. Ser.: Phys.-Math. 2004, N4, 427-432.

25. Bystryantseva, A., Khetselius, O.Yu., Dubrovskaya, Yu., Vitavetskaya, L.A., Berestenk, A.G. Relativistic theory of spectra of heavy pionic atomic systems with account of strong pion-nuclear interaction effects: ${ }^{93} \mathrm{Nb},{ }^{173} \mathrm{Yb},{ }^{181} \mathrm{Ta},{ }^{197} \mathrm{Au}$. Photoelectronics. 2016, 25, 56-61.

26. Buyadzhi, V., Zaichko, P., Antoshkina, O., Kulakli, T., Prepelitsa, P., Ternovsky, V., Mansarliysky, V. Computing of radiation parameters for atoms and multicharged ions within relativistic energy approach: Advanced Code. J. Phys.: Conf. Ser. 2017, 905(1), 012003.

27. Buyadzhi, V., Kuznetsova, A., Buyadzhi, A., Ternovsky, E.V., Tkach, T.B. Advanced quantum approach in radiative and collisional spectroscopy of multicharged ions in plasmas. Adv. in Quant. Chem. 2019, 78, 171-191.

28. Khetselius, O.Yu., Lopatkin, Yu.M., Dubrovskaya, Yu.V, Svinarenko, A.A. Sensing hyperfine-structure, electroweak interaction and parity non-conservation effect in heavy atoms and nuclei: New nuclear-QED approach. Sensor Electr. and Microsyst. Techn. 2010, 7(2), 11-19.

29. Glushkov, A.V., Khetselius, O.Yu., Svinarenko, A., Buyadzhi, V. Methods of computational mathematics and mathematical physics TES: Odessa, 2015

30. Ignatenko, A.V., Svinarenko, A.A., Prepelitsa, G.P., Perelygina, T.B. Optical bi-stability effect for multi-photon absorption in atomic ensembles in a strong laser field. Photoelectronics. 2009, 18, 103-105.

PACS 31.15.A-; 32.30.-r

A. S. Chernyshev, O. L. Mykhailov, A. V. Tsudik, I. S. Cherkasova

\section{RELATIVISTIC THEORY OF CALCULATION OF E1 TRANSITION AMPLITUDES, AND GAUGE INVARIANCE PRINCIPLE}

Summary. The combined relativistic energy approach and relativistic many-body perturbation theory with the zeroth order Dirac-Kohn-Sham one-particle approximation are used for estimating the energies and the E1 radiative transitions amplitudes (oscillator strengths) for the low-excited states of the francium. The comparison with available theoretical and experimental (compillated) data is performed. The important point is linked with an accurate accounting for the complex exchange-correlation (polarization) effect contributions and using the optimized one-quasiparticle 
representation in the relativistic many-body perturbation theory zeroth order that significantly provides a physically reasonable agreement between theory and precise experiment.

Key words: relativistic theory, radiative transitions, francium

PACS 31.15.A-; 32.30.-r

А. С. Чернышиов, А. Л. Михайлов, А. В. Цудик, И. С. Черкасова

\section{РЕЛЯТИВИСТСКАЯ ТЕОРИЯ РАСЧЕТА ПЕРЕХОДНЫХ АМПЛИТУД Е1 ПЕРЕХОДОВ И ПРИНЦИП КАЛИБРОВОЧНОЙ ИНВАРИАНТНОСТИ}

Резюме. Комбинированный релятивистский энергетический подход и релятивистская многочастичная теория возмущений с дирак-кон-шэмовским одночастичным нулевым приближением используются для для вычисления энергий и амплитуд E1 радиационных переходов (сил осцилляторов) для низко возбужденных состояний франция. Проведено сравнение с имеющимися теоретическими и экспериментальными данными. Важный момент связан с аккуратным учетом вкладов сложных многочастичных обменных корреляционных (поляризационных) эффектов и с использованием оптимизированного одноквазичастичного представления в нулевом приближении релятивистской многочастичной теории возмущений, что определяет определенное согласие теории и эксперимента.

Ключевые слова: релятивистская теория, радиационные переходы, франций

PACS 31.15.A-; 32.30.-r

О. С. Чернишов, О. Л. Михайлов, А. В. Цудік, І. С. Черкасова

\section{РЕЛЯТИВІСТСЬКА ТЕОРІЯ РОЗРАХУНКУ АМПЛІТУД Е1 ПЕРЕХОДІВ І ПРИНЦИП КАЛІБРУВАЛЬНОЇ ІНВАРІАНТНОСТІ}

Резюме. Комбінований релятивістський енергетичний підхід i релятивістська багаточастинкова теорія збурень 3 дірак-кон-шемівським одночастинковим наближенням нульового порядку використовуються для обчислення енергій та амплітуд Е1 радіаційних переходів (сил осцилляторов) для низько збуджених станів францію. Проведено порівняння 3 наявними теоретичними і експериментальними даними. Важливий момент пов'язаний 3 акуратним урахуванням вкладів складних багаточасткових обмінних кореляційних (поляризаційних) ефектів і з використанням оптимізованого одноквазічастічного уявлення в нульовому наближенні релятивістської багаточастинкової теорії збурень, що визначає певну згоду теорії та експерименту.

Ключові слова: релятивістська теорія, радіаційні переходи, францій 